\title{
激光显示技术发展的现状和趋势
}

\author{
高伟男，许祖彦，毕勇，袁园 \\ (中国科学院理化技术研究所, 北京 100190)
}

摘要：随着信息技术的不断发展, 信息化时代已经来临, 显示作为人机信息交互环节最重要的信息输出终端, 已经遍及人们 生活的各个方面。当今时代显示技术层出不穷, 都以实现超高分辨率、大尺寸、真色彩的极致画面显示, 满足人们追求极致 美好视觉的需求为发展方向, 目标就是实现高保真图像再现。激光显示是采用三基色激光作为显示光源的新型显示技术, 可 以实现双高清 (几何/颜色)、大色域、高观赏舒适度的 “冲击人眼视觉极限” 高保真图像再现, 被视为显示产业转型升级 的重要方向之一。本文立足新形势下显示产业自主可控安全发展的需求, 详细论述了激光显示技术和产业特点, 梳理和总结 了本领域发展现状、趋势和短板问题, 凝练出激光显示产业发展路线图, 建议我国应抓住这一大好机遇, 加大关键核心技术 攻关，自主可控加速将激光显示产业发展壮大，支撑下一代显示产业的高端市场，并开拓出激光显示规模产业集群。

关键词：激光显示; 双高清; 大色域; 高观赏舒适度

中图分类号: TN27 文献标识码：A

\section{Present Development and Tendency of Laser Display Technology}

\section{Gao Weinan, Xu Zuyan, Bi Yong, Yuan Yuan}

(Technical Institute of Physics and Chemistry, Chinese Academy of Sciences, Beijing 100190, China)

\begin{abstract}
Display device is the terminal of information display in interactions between human and machine and is expanding to all aspects of people's lives in the information age. Nowadays, all display technologies are developing toward high-fidelity images with ultra-high resolution, large screen, and true color, to satisfy people's needs for perfect visual senses. Laser display technology uses tricolor laser as the display light source and can realize the reproduction of high-fidelity images with dual high definition (geometry/ color), large color gamut, and high viewing comfort, which approaches the limit of human vison. Laser display is thus regarded as one of the significant directions for transformation and upgrading of the display industry. In this study, the technical and industrial characteristics of the laser display technology are analyzed in details based on the requirements of the industry for independent, controllable, and safe development. The current development situation, development trend, and key problems are summarized in the laser display industry. The development roadmap of China's laser display industry is proposed. China should make more effects in breakthroughs of key technologies to grow its laser display industry in an independent and controllable manner. It also should support the high-end market of the next-generation display industry and build industrial clusters for laser display.
\end{abstract}

Keywords: laser display; dual high definition; large color gamut; good visual comfort

收稿日期 : 2020-03-26; 修回日期 : 2020-04-20

通讯作者: 许祖彦, 中国科学院理化技术研究所研究员, 中国工程院院士, 主要从事激光技术及应用研究; E-mail: zyxu@mail.ipc.ac.cn 资助项目：中国工程院咨询项目“我国激光技术与信息应用 2035 发展战略研究” (2018-XZ-27)

本刊网址： www.engineering.org.cn/ch/journal/sscae 


\section{一、前言}

信息技术是有关信息的采集、储存、处理、传 输、显示等各信息链关键技术的总称, 是我国战略 性新兴产业。显示作为信息技术的重要组成和信息 链的终端人机界面, 应用领域已遍及工业、交通、 通信、教育、航空航天、卫星遥感、娱乐、医疗等 日常生活各方面, 是信息产业的一个重要支柱。随 着第五代移动通信技术 $(5 \mathrm{G})$ 、大数据、人工智能 等未来信息技术的发展和融合, 显示将向泛在、融 合、智能和绿色方向发展, 需求增长空间巨大。

激光显示是以红 $(R)$ 、绿 $(\mathrm{G})$ 、蓝 $(B)$ 三基色 (或 多基色）激光为光源的新型显示技术和产品, 通过 控制三基色激光强度比、总强度和强度空间分布即 可实现彩色图像显示 [1], 如图 1 所示。由于激光 具有方向性好、单色性好和亮度高等三个基本特性, 用于显示可实现 “冲击人眼极限” 的大色域、双高 清 (几何、颜色) 的高保真视频图像再现, 被国际 业界视为 “人类视觉史上的革命”, 是继黑白显示、 彩色显示、数字显示之后的新型显示技术 (见图 2), 《〈中国制造 2025〉重点领域技术路线图（2015 年 版)》已将其列入发展重点, 是显示产业转型升级 的重要战略方向 [2]。

\section{二、激光显示技术的特点}

研究表明，人类获取的信息中有 $70 \%$ $80 \%$ 来 自于视觉, 显示作为人机界面终端, 最终是要满足 人眼观看、观赏等需求和实现这些功能。激光显示 作为新一代显示技术, 在继承了数字显示技术所有 优点的基础上, 还具有以下本征优势, 可真正实现

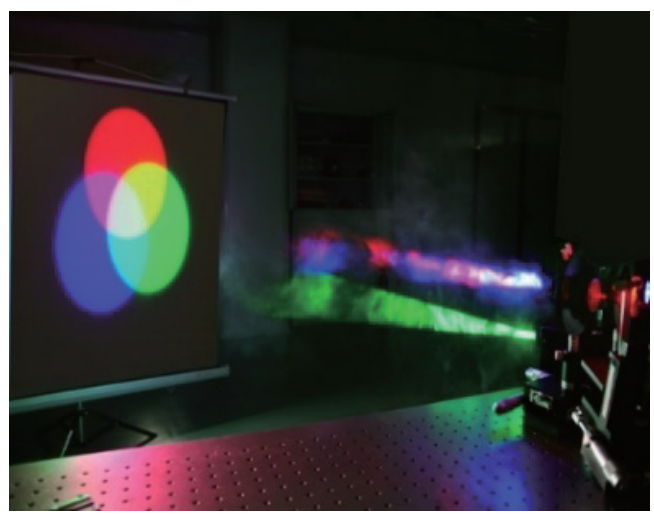

图 1 红、绿、蓝三基色激光合成白光实验
高保真图像再现。

\section{（一）几何／颜色双高清}

几何高清即线分辨率。人眼生物学和视光学原 理表明, 人眼极限分辨力约为 $1^{\prime}$, 人眼清晰视场为 横向 $35^{\circ}$ 、纵向 $20^{\circ}$, 余光视场横向 $120^{\circ}$ 、纵向 $60^{\circ}$ [1], 因此人眼可观察到超高分辨率图像以及 丰富和精细的色彩。激光的方向性好，其发散角 小，易实现 $4 \mathrm{~K} 、 8 \mathrm{~K}$ 甚至更高的（全屏）显示 分辨率。

颜色高清即颜色数。激光显示最突出的特点 就是激光光谱很窄, 称之为线状光谱, 谱宽小于 $5 \mathrm{~nm}$, 而其他显示光源基本为带状光谱, 谱宽约为 30 40 nm, 如图 3 所示。由于谱宽太宽, 三基色 光谱在颜色混合时很多精细的颜色重叠人眼已经分 辨不出, 无法在终端很好地展现出来。而采用小于 $5 \mathrm{~nm}$ 光谱宽度的三基色激光作为光源时, 颜色的色 纯度很高, 可以完全实现 12 bit 颜色数编码不重叠, 实现传统显示的 500 倍以上的大颜色数显示, 更能 反映自然界的真实色彩 [3 6]。

\section{（二）大色域}

现有的彩色显示设备, 其红、绿、蓝三基色光 源为带谱, 在色度图中, 其色域只覆盖了人眼所能 识别颜色的一小部分, 因而不能再现饱和度很高的 颜色。激光的单色性好, 其光谱为线谱, 可在色度 图上形成超大色域, 如图 4 所示, 颜色更加鲜艳, 颜色表现能力是传统显示器的 2 3 倍, 拥有无与伦 比的颜色再现能力。

\section{（三）高观赏舒适度}

激光显示采用反射式成像，与自然万物反射光

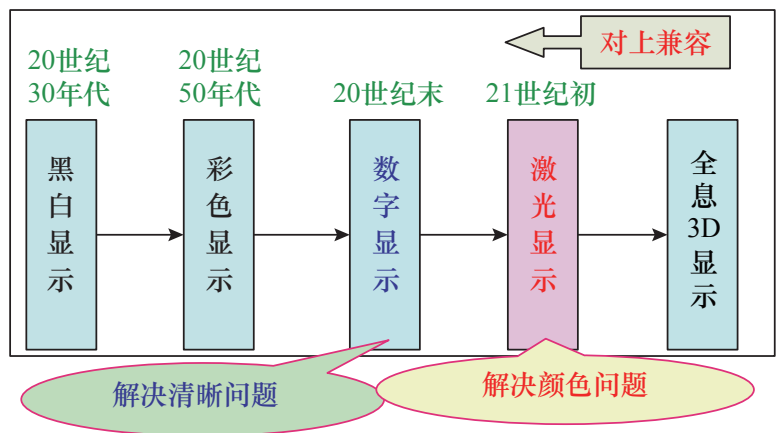

图 2 显示技术发展路线图 


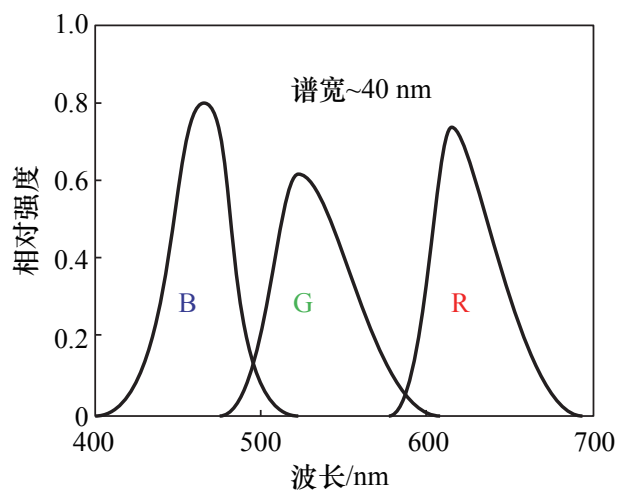

(a) 传统光源谱图——宽带非相干光源

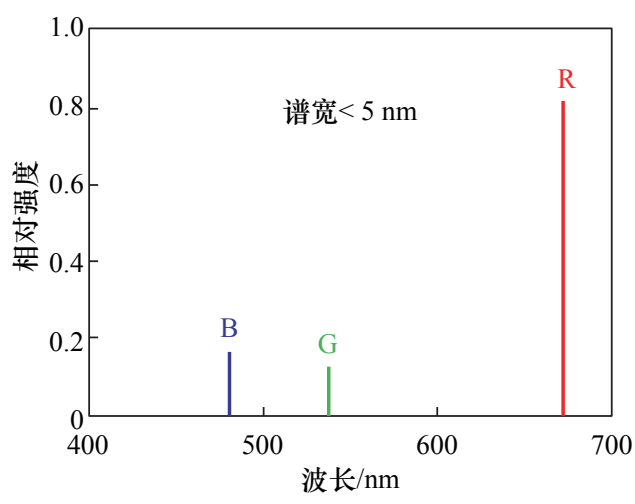

(b) 激光光源谱图——窄带相干光源

图 3 传统显示的波长与激光显示的波长对比

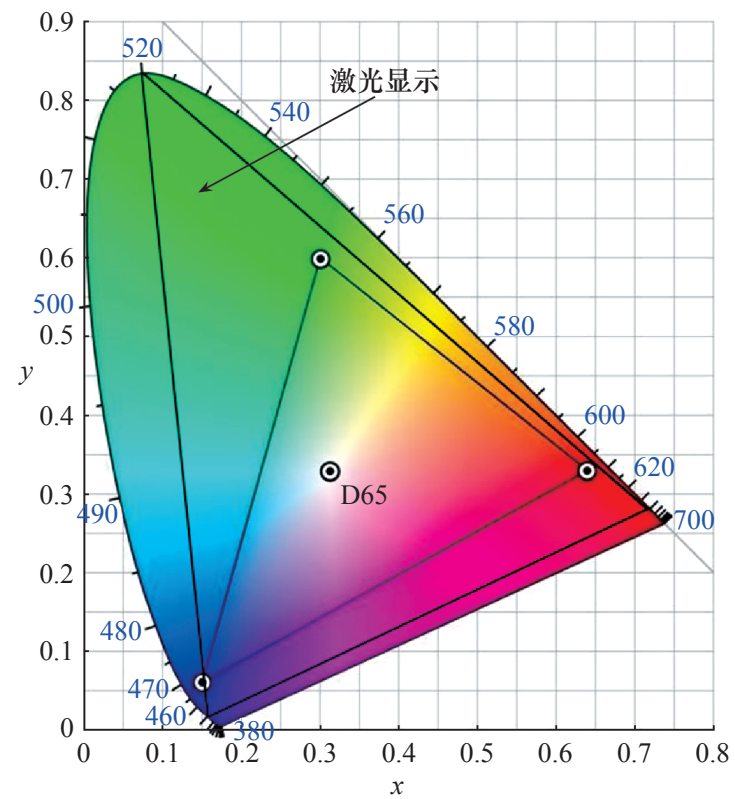

图 4 不同显示技术在色度图中实现的色域

成像进入人眼原理相同, 光线经屏幕反射至人眼, 光线柔和不刺眼; 同时激光显示工作原理决定了其 像素与发光面积相同, 且像素与像素之间无边缘效 应, 过渡平缓, 因此观看舒适度高。中国电子技术 标准化研究院赛西实验室与北京协和医院进行观看 舒适度测试, 结果显示激光显示是良好舒适性的显 示产品 [7]。

综上所述, 在技术上激光显示具有很多优点: 光源谱宽窄 $(\sim 5 \mathrm{~nm})$, 可以实现 12 bit 颜色灰阶编 码不重叠; 激光波长可控, 依据 1952 年美国国家 电视标准委员会制定的彩色电视广播标准（简称 “NTSC 标准”), 可构成 150\% NTSC 以上超大色域;
激光亮度高, 且可精确控制在人眼最佳视觉感知区 (8 K 几何高清)；激光色温精确可调，极易实现超 大屏幕 (百平米级) 无缝拼接显示; 与全息技术结 合, 可再现物光波长(颜色)、振幅 (强度) 和相位 (立 体) 的全部信息, 实现真三维显示。激光显示是目 前唯一能够实现 BT.2020 超高清国际显示标准的显 示技术 [8], 可满足人类追求美好视觉效果的极致 需求。

同时, 激光显示还兼具轻薄、低成本、绿色 制造等特点：功耗比同尺寸液晶电视节能 $50 \%$, 寿命可达 $2 \times 10^{4} \mathrm{~h}$ 以上, 节能环保; 产品体积小、 重量轻、价格低, 100 in 激光电视质量约为 $20 \mathrm{~kg}$ (同尺寸液晶电视质量约为 $150 \mathrm{~kg}$ ), 容易投放于 电梯和进入寻常百姓家; 激光显示采用反射式成 像, 观看舒适度好; 激光显示属于绿色制造产业, 不需要大型投资规模, 相比于传统平板显示在高 世代面板的巨大投资（显示面板领域累计投入超 过 1.2 万亿元), 激光显示制程工艺简单, 符合新 型显示柔性、便携、低成本、高色域、高光效的 发展趋势。

因此, 激光显示可兼顾观赏 / 娱乐和信息两大 市场, 将是下一代电视机、电影机、超大屏幕显 示产品的主流。激光显示作为新型显示技术的代 表, 与我国《〈中国制造 2025〉重点领域技术路线 图 (2015 年版)》和《超高清视频产业发展行动计 划（2019-2022 年)》等国家重大战略高度契合, 已经成为我国影响国计民生以及后续发展的优势产 业, 是维护国家产业安全、体现国家信息技术智能 化水平、促进产业转型升级的重要战略产业。 


\section{三、国内外激光显示技术现状}

\section{（一）国外激光显示发展情况}

国外激光显示强国, 如日本、韩国、美国早 在 20 世纪 90 年代就开始布局激光显示关键技术研 究, 先后通过美国能源部计划、日本科技基本计划 等国家项目予以引导, 支持相关企业开展攻关。其 中日本日亚、三菱集团等公司已投入约 32 亿美元 研发三基色半导体激光器 ( $\mathrm{LD}$ ), 其中磷化铟 ( InP) 红光 $\mathrm{LD}$ 单管功率可达 $750 \mathrm{~mW}$, 氮化镓 ( $\mathrm{GaN}$ ) 蓝、绿光 $\mathrm{LD}$ 分别实现超过 $4 \mathrm{~W}$ 和 $1 \mathrm{~W}$ 的单管输 出功率, 寿命超过 $2 \times 10^{4} \mathrm{~h}$, 技术经济指标国际领 先; 美国德州仪器 ( TI )、日本索尼集团基本垄断 $2 \mathrm{~K} / 4 \mathrm{~K}$ 分辨率的反射式数字微镜 (DMD)、反射 式硅基液晶 (LCOS) 激光显示超高清图像处理芯 片; 日本理光集团、DNP 印刷株式会社等企业在 超高清镜头以及超大尺寸菲涅尔光学膜片等技术 领域处于世界领先。

在整机关键技术方面, 日本由政府组织企业和 研究机构共同大力研发激光显示技术, 索尼集团、 松下电器产业株式会社、三菱集团、精工爱普生等 公司支持, 制定了 “xvYCC” 大色域显示标准, 并 在全球范围进行产业链整合, 维护日本下一代显示 技术的竞争优势。三菱电机成功研制激光背投电 视、65 in 激光电视、3D 激光电视; 精工爱普生公 司推出了基于 $3 \mathrm{LCD}$ 的激光前投影样机。在欧美, 多家公司根据应用场景积极开发激光显示整机, 德 国耶拿光学集团公司开发了应用于天文馆等特殊市 场的激光显示产品; 美国 Evans \& Sutherland 公司 利用大屏幕激光显示系统开发飞行员仿真视景平 台, 用于战斗机飞行员训练; B arco 公司推出了 $4 \mathrm{~K}$ 分辨率 $60000 \mathrm{~lm}$ 的激光投影机, 2019 年展示了 基于 RGB 激光光源、矩形阵列像素数字微镜器件 (TRP DMD)、 $4 \mathrm{~K}$ 分辨率、98.5\% REC2020 色域的 激光电影放映机等; 2014 年科视数字投影系统公司 (Christie) 展示了使用六原色 $(6 \mathrm{P})$ 的 $4 \mathrm{~K}$ 分辨率 $3 \mathrm{D}$ 激光放映系统, 2019 年在 BIRTV 展示了 $4 \mathrm{~K}$ 分 辨率、 $120 \mathrm{~Hz}$ 的高帧率的 RGB 电影放映机。

\section{（二）国内激光显示发展现状}

国内激光显示发展与国外基本同步, 已经从
过去的跟跑发展到总体并跑、产业规模领跑的阶 段。中国科学院研究团队一直是国内从事激光显示 领域的领军团队, 在国内最早开展激光显示技术研 发。20 世纪 70 年代中国科学院物理研究所等单位 实现了基于气体激光光源的扫描式激光显示样机开 发。在国家高技术研究发展计划 (863 计划) 和中 国科学院创新工程计划支持下, 中国科学院许祖彦 在 2003 年研制成功国内首台激光投影显示原理样 机; 2005 年推出 65 in、84 in、140 in、200 in 激光 电视样机 (见图 5); 2006 年 1 月通过了工业和信 息化部和中国科学院联合鉴定, 鉴定认为总体水平 世界先进, 色域覆盖率等关键技术国际领先, 并拥 有多项核心技术发明专利为代表的知识产权, 与国 际同期完成了激光显示研究阶段, 加速推动了激光 显示从样机走向实用化的进程; 同年, 许祖彦提出 了以红、绿、蓝三基色半导体激光 (LD) 为核心 的产业发展路线图, 并在 2015 年率团队研制成功 国际首台 100 in 三基色 LD 电视样机, 证明了激光 显示技术实现产业化的可行性, 随后建成三基色激 光显示生产示范线, 初步打通了激光显示材料、器 件、整机到产业示范的创新链。

近年来, 国内激光显示技术和产业发展迅速, 从 “十五” “十一五” “十二五” 的 863 计划、科技 支撑计划, 到 “十三五” 的重点研发计划, 激光显 示关键材料、器件与应用技术逐步取得突破, 尤其 是在激光显示产业核心一三基色 $\mathrm{LD}$ 以及整机设 计制造方面进展明显, 目前国内红光 LD 单管功率 可达 $2 \mathrm{~W}$ (寿命超过 $1 \times 10^{4} \mathrm{~h}$ ), 蓝光 LD 单管最大 输出功率为 $2.8 \mathrm{~W}$ (寿命已超过 $5000 \mathrm{~h}$ ), 绿光 LD 最大输出功率达到 $500 \mathrm{~mW}$; 整机方面, 我国在高 功率激光模块、散斑抑制和集成制造关键技术方面 已经形成多项核心专利技术, 总体已达国际领先水 平。国家出台一系列相关政策及项目支持, 极大地 推动了骨干企业在激光显示的投入和产业的快速发 展, 国内包括海信视像科技股份有限公司、四川长 虹电器股份有限公司、杭州中科极光科技有限公司、 深圳光峰科技股份有限公司、 TCL 科技集团股份有 限公司等数十家传统家电企业和互联网企业, 围绕 激光显示全链条开展产业布局, 2019 年国内激光显 示产值已经超过 125 亿元, 近几年年复合增长率接 近 100\%, 产业规模达国际领先水平。 


\section{四、激光显示的发展趋势分析和挑战}

激光显示技术要走向产业应用, 亟待解决红 绿蓝三基色光源、超高清视频图像技术、配套关 键材料与器件、总体设计与集成这四大关键技术 (见图 6), 同时系统布局激光显示专利池和国际标 准, 有望打造具有自主知识产权的激光显示产业生 态体系, 抓住显示产业转型升级重大机遇, 实现我 国显示产业从大国到强国的跨越式发展。

\section{（一）三基色激光光源}

纵观激光显示几十年来的发展历程, 实际上 驱动着激光显示产业不断发展的核心推动力就是激 光光源, 也是激光显示实现高画质图像再现的核心 竞争力。如图 7 所示, 激光显示光源经历了从气体 激光器（体积大、耗电大、寿命短、不易实用化）、 全固态激光器 (结构复杂、效率低、难以消散斑), 目前已发展到三基色 LD 光源为代表的阶段。

三基色 $\mathrm{LD}$ 光源，与其他相干 / 非相干光源相

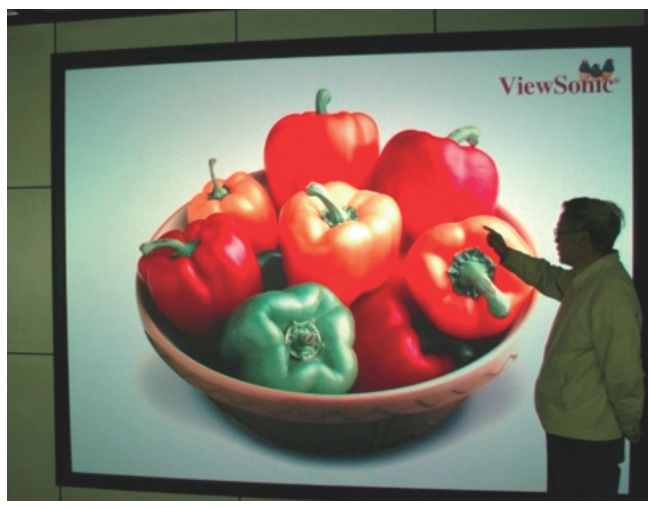

图 52005 年国内首台激光全色投影显示样机
比, 具有直接电激发、高效率、高偏振度、长寿 命、高可靠、小型化、频域/空域/时域综合参数 易于调控等优势, 更重要的是, LD 具有可用半导 体制造工艺实现大规模量产降低成本的独到优势, 支撑激光显示实现高性价比, 可进入寻常百姓家。 $\mathrm{LD}$ 是激光显示产业化的最佳光源。目前国内三基 色 $\mathrm{LD}$ 材料器件已经取得了重大进展, 红光 $\mathrm{LD}$ 单 管功率可达 $2 \mathrm{~W}$ (寿命超过 $1 \times 10^{4} \mathrm{~h}$ ), 蓝光 $\mathrm{LD}$ 单管最大输出功率为 $2.8 \mathrm{~W}$ (寿命已超过 $5000 \mathrm{~h}$ ), 绿光 $\mathrm{LD}$ 最大输出功率达到 $500 \mathrm{~mW}$, 已经接近了 实用化水平。

\section{（二）超高清视频图像技术}

显示已经进入超高分辨率时代, $8 \mathrm{~K}$ 超高清电 视技术全球竞争已经展开，工业和信息化部等多部 门印发《超高清视频产业发展行动计划（20192022 年)》, 预示着我国 2022 年要实现超高清视频 生态体系建设。激光显示作为超高清显示技术的代 表, 亟待解决 $4 \mathrm{~K} / 8 \mathrm{~K}$ 超高分辨率显示芯片、超高 清视频图像的获取 / 存储 / 处理 / 传输、人眼生物 学特征和视觉心理特性等关键技术。在超高清视频 标准方面我国进展较快, 自主研究制定自主数字视 音频编解码技术标准。同时, 新一代信息技术比如 $5 \mathrm{G}$ 、大数据、云计算技术的普及，使激光显示视频 图像信息的高速率、大带宽需求成为可能, 支撑超 高清电视信号进入寻常百姓家。

\section{（三）激光显示配套材料与器件}

超高分辨光学成像镜头、高增益光学屏幕、新 型匀光整形材料、智能驱动 / 显示芯片等激光显示

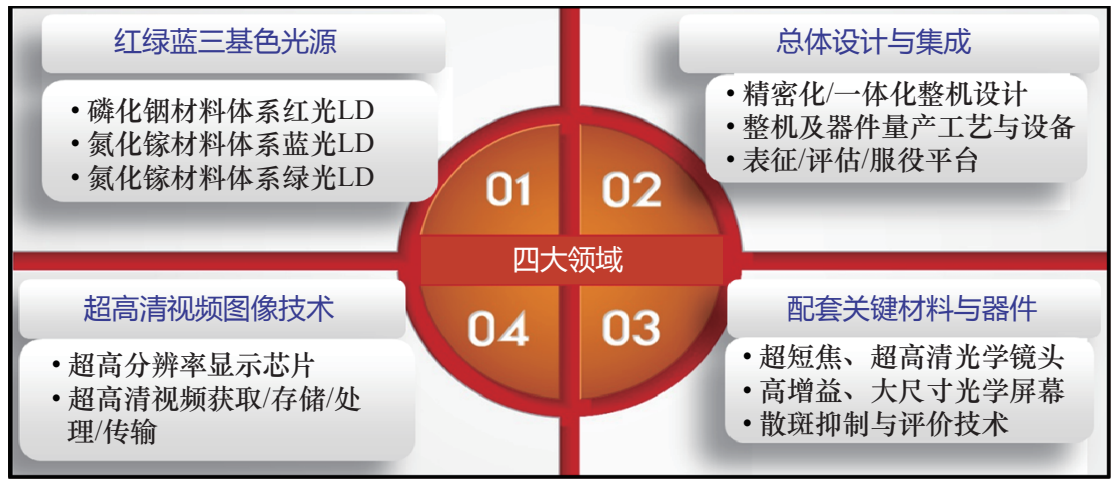

图 6 激光显示四大关键技术 


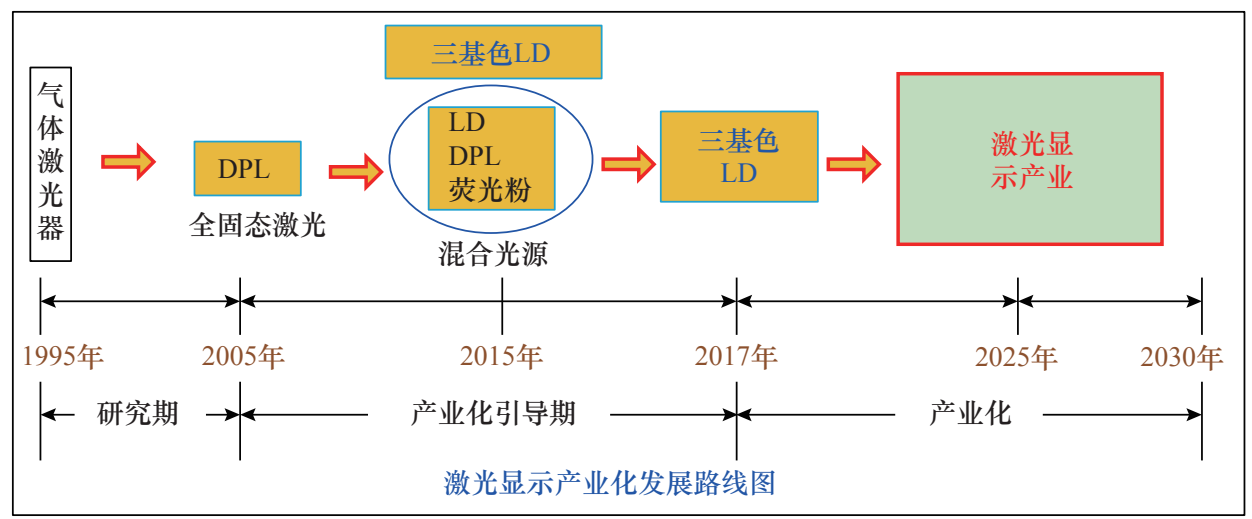

图 7 激光显示产业发展路线

配套关键材料与器件, 是激光显示生态体系必不可 少的一环。我国已掌握了超短焦镜头设计和制造能 力, 初步实现了镜头、光机在内的 $4 \mathrm{~K}$ 光学引擎的 批量生产; 在高性能菲涅尔光学屏幕方面, 持有多 项核心设计 / 制造专利, 实现了超大尺寸光学屏幕 生产示范; 在散斑抑制技术方面, 国内已有很多专 利技术基础, 提出多手段消散斑技术和评价方法, 加速了其实用化进程 [9 12]。另外, 关键材料生长 和加工设备如菲涅尔模具大型 $\mathrm{CNC}$ 加工设备等也 亟需解决依赖进口的问题。

\section{（四）激光显示整机总体设计与集成}

在激光显示整机方面, 需要重点开展超高清、 大色域整机设计、关键器件集成、高良率量产工 艺、可靠性测试以及相应的标准等产业化应用技 术研究, 具体包括光学设计、图像处理、整机鲁 棒性和寿命检测 / 分析及优化对策研究 [13], 以 及规模量产工艺与设备研究, 以整机应用需求为 牵引, 带动激光显示关键材料器件快速走向应用, 从而建成完备的设计、材料生长、器件制备、整 机集成到产业应用的全链条创新体系。

\section{五、我国激光显示领域存在的主要问题}

\section{（一）关键材料器件对外依存度高, 产业自主发展 存在安全隐患}

我国激光显示产业已达世界领先, 但是不应忽 视的是, 红绿蓝三基色激光器、超高清成像芯片等 核心材料器件尚未实现自主可控, 存在 “卡脖子”
问题, 产业发展存在重大隐患。

\section{（二）研究与产业应用衔接度低, 未形成良好的评 价体系引导核心技术发展}

激光显示关键材料器件的研发和攻关与整机应 用衔接度不高, 围绕关键材料器件的综合性能测试 和应用技术评价体系尚未完善, 稳定性、寿命、装 备等技术经济指标与国外相比尚有很大差距, 导致 创新链上下游未完全贯通。

\section{（三）产业集中度不高，产业生态体系尚不完善}

目前国内多家研究机构和企业从事激光显示技 术研发和产业攻关, 但是缺乏统一部署, 研发力量 相对薄弱, 资金投入分散, 很多企业追逐短平快项 目而缺少长远规划, 导致目前产业集中度不高, 没 有围绕理论、关键技术、创新应用等层面构建完善 的技术、人才和知识产权的协同创新体系。

\section{六、政策建议}

(一) 加强顶层设计, 建立国家引导、资源整合产 业发展新机制

激光显示作为我国自主创新的新一代信息技 术, 具有产业规模大、经济带动性强、技术集成度 高的特点, 需要加强对激光显示技术及产业未来发 展趋势研判, 对产业发展进行规划和顶层设计, 建 立国家引导、投入稳定增加的研发新机制, 各级政 府能够在保持产业支持政策延续性的同时, 加大对 国内具有自主创新能力与核心技术企业的政策支持 
力度, 与企业自主创新形成合力, 形成社会多元投 入支持的材料、技术、器件到产业的良好局面。

\section{（二）加大关键核心技术研发力度，布局颠覆性技 术，提高本领域自主创新能力}

激光显示具有超高清、大色域、高亮度、高观 赏舒适度等优势, 我国已经在激光电视、激光影院 等领域实现了产品化应用，并逐渐形成高端显示产 品门类, 产业规模已达国际领先, 但长期遭受光源 芯片、显示芯片等核心技术被国外垄断，成为产业 发展的 “卡脖子” 问题。为了继续保持在激光显示 领域的技术和产业优势，应通过 “政产学研” 联合 创新, 注重应用驱动, 加大三基色 $\mathrm{LD}$ 光源和超高 清显示芯片的国产化研究, 在短期内补足短板; 布 局全息真三维显示等激光显示前瞻性技术，从而在 远期形成先发优势。

\section{（三）建设专利池、制定标准与规范，为提高我国 激光显示技术和产品核心竞争力提供支撑}

知识产权是自主可控发展我国激光显示产业的 导引和抓手，是规避贸易壁垒、将产品推向全球的 最有效办法。我国在激光显示知识产权方面有较好 的积累和储备, 现有专利超过 7000 项, 主导和参 与了多项国际标准的制定, 知识产权体系初步建立。 未来应重点围绕激光显示核心材料器件开展全链条 布局，突破核心技术制约，建设完整的激光显示专 利池、技术标准和规范，加速推动我国激光显示技 术及产品全球化，取得国际话语权。

（四）建设激光显示创新平台，培育产业生态，构 建产业体系，实现可持续发展

围绕激光显示全产业链, 打造建立激光显示创 新平台，以关键材料为基础，以激光显示制造技术 为核心, 上承材料/装备, 下启整机/终端, 加强 体现激光显示技术优势的视频内容产品和高速信息 处理技术研发，解决产业化共性关键技术，布局前 瞻性技术，培养创新团队和人才，最终全面形成具 有自主知识产权的覆盖激光显示上下游的激光显示 产业生态体系, 支撑产业聚集, 实现我国显示产业 由大到强的跨越式发展。

\section{参考文献}

[1] Kishore V C, Erdem E, Hakan U. Laser-based displays: A review [J]. Applied Optics, 2010, 49(25): F79.

[2] 许祖彦. 光电子晶体与全固态激光器及其应用一一光电子技术 发展的一个重大方向 [J]. 中国工程科学, 1999, 1(2): 72-77.

Xu Z Y. Optoelectronic crystals, all-solid-state lasers and their applications__ An Important course of optoelectronics R \&D [J]. Strategic Study of CAE, 1999, 1(2): 72-77.

[3] 荆其诚, 焦书兰, 喻柏林, 等. 色度学 [M]. 北京: 科学出版社, 1979

Jing Q C, Jiao S L, Yu B L, et al. Chromaticity [M]. Beijing: China Science Publishing \& Media Ltd., 1979.

[4] 毕勇. 激光全色显示技术 [J]. 新材料产业, 2009 (4): 76-71.

Bi Y. The full color laser display [J]. Advanced Material Industry, 2009 (4): 76-71.

[5] 许祖彦. 大色域显示——新一代显示技术 [J]. 物理, 2010, 39(4): 227-231.

Xu Z Y. Large color gamut display_ _ The new generation of display technique [J]. Physics, 2010, 39(4): 227-231.

[6] 许祖彦. 激光显示一一新一代显示技术 [J]. 激光与红外, 2006, 36(B09): 737-741.

Xu Z Y. Laser displays_— New display technology for next generation [J]. Laser \& Infrared, 2006, 36(B09): 737-741.

[7] 许祖彦, 毕勇, 张文平. 激光显示是我国新型显示技术发展主 流 [J]. 电子科学技术, 2019 (1): 53-61.

Xu Z Y, Bi Y, Zhang W P. Laser displays: Developing trend of Chinese new display technolog [J]. Electronic Science \& Technology, 2019 (1): 53-61.

[8] 国际电联无线电通信部门. R12-SG06 BT.2020-2-2015 超高清 电视系统节目制作和国际交换的参数数值 [S]. 日内瓦: 国际 电信联盟, 2017: 2 .

International Telecommunication Union. R12-SG06 BT.2020-22015 Recommendation parameter values of program production and international exchange in UHD TV system [S]. Geneva: International Telecommunication Union, 2017: 2.

[9] Yuan Y, Fang T, Sun M Y, et al. Speckle measuring instrument based on biological characteristics of the human eyes and speckle reduction with advanced electromagnetic micro-scanning mirror [J]. Laser Physics, 2018, 28(7): 5002-5007.

[10] Yuan Y, Wang D Z, Zhou B J, et al. High luminous fluorescence generation using Ce:YAG transparent ceramic excited by blue laser diode [J]. Optical Materials Express, 2018, 8(9): 2760-2767.

[11] Wang D Z, Yan B X, Bi Y, et al. Three-wavelength green laser using intracavity frequency conversion of $\mathrm{Nd}: \mathrm{Mg}: \mathrm{LiTaO}_{3}$ with a MgO:PPLN crystal [J]. Applied Physics B, 2014 (117):1117-1121.

[12] Wang D Z, Sun D H, Kang X L, et al. Periodically poled self-frequency-doubling green laser fabricated from $\mathrm{Nd}: \mathrm{Mg}: \mathrm{LiNbO}_{3}$ single crystal [J]. Optics Express, 2015, 23(14): 17727-17738.

[13] 郁道根. 工程光学(第四版) [M]. 北京: 机械工业出版社, 2016. Yu D G. Engineering optics (fourth edition) [M]. Beijing: Mechanical Industry Press, 2016. 